$\mathbb{T}$ periodica polytechnica

Civil Engineering

$53 / 1$ (2009) $3+8$

doi: 10.3311/pp.ci.2009-1.01

web: http://www.pp.bme.hu/ci

(c) Periodica Polytechnica 2009

RESEARCH ARTICLE

\section{Stochastically generated finite element beam model for dental research}

\author{
Ilona Éva Lakatos / Imre Bojtár
}

Received 2008-12-05, accepted 2009-03-09

\section{Abstract}

Dental implantation is currently the most commonly used and physiologically the most favourable procedure for tooth replacement in dental surgery. Implants can have either advantageous or destructive effect on the surrounding bone, depending on several physiological, material and mechanical factors. The most general method for estimating the biomechanical behaviour of the bone is Finite Element Analysis. The microstructural conformation of the trabecular bone - which can be modelled by the means of converting computed tomography images into micro finite element models or in a stochastic way - influences the overall mechanical properties of the bone tissue. To avoid the use of computer tomography imaging and to create a repeatable and variable finite element model, a stochastically generated beam structure was accomplished that possesses the geometrical and mechanical microstructural properties - obtained from literature - of the trabecular bone substance of an average man from the edentulous mandibular region. The finite element beam model was submitted to compression tests, and the macrostructural elastic properties were computed from the result data obtained by the means of Finite Element Analysis. Several attempts have been made to achieve the possibly most accurate elastic properties. Considering the shell behaviour of the trabeculae by dividing each beam into three parts with different elastic properties proved to be the most effective and the most suitable for further investigations, in which different types of loading and the finite element model of an implant embedded in the bone is planned to be used.

\section{Keywords}

Dental research · Bone mechanics - Trabecular bone · Finite element analysis

\section{Ilona Éva Lakatos}

\section{Imre Bojtár}

Department of Structural Mechanics, BME, H-1111 Budapest, Múegyetem rkp. 3., Hungary

\section{Introduction}

The biomechanical behaviour of a dental implant (Fig. 1) - artificial tooth root replacement that is to hold the prosthesis and to fasten to the bone [2] - plays an important role in its functional longevity inside the bone. Occlusal forces affect the bone surrounding an oral implant. The following study deals with especially the trabecular bone substance surrounding dental implants considering the microscopic conformation of the bone.

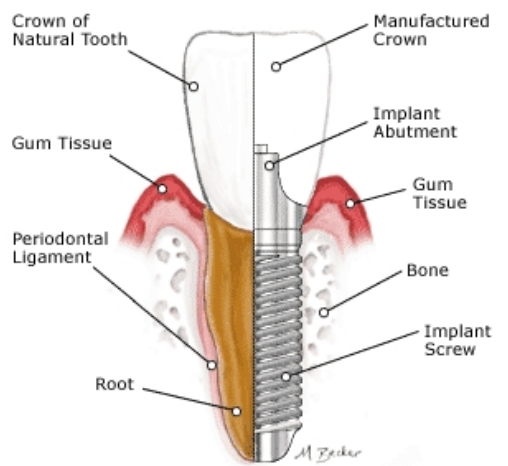

Fig. 1. The parts of a screw type dental implant [www.pujadentalgroup.com/images/dental-implant.gif]

Application of finite element analysis has become an indispensable method for estimating mechanical behaviour - stress and strain distributions under a certain load - of the cortical and cancellous bone surrounding dental implants, since being applicable in vivo. These numerical experiments have their importance in making the implantation the possibly most secure, reliable and efficient, and the lifetime of the implant the longest conceivable, by finding the most favourable thread formation, surface, material, etc. In finite element analysis of the bone several assumptions need to be made, that influence the accuracy of the results. Recently the geometry of the implant can be modelled accurately, the assumptions are made in the aspect of the bone and bone-implant interface - where optimal osseointegration is assumed, which is acceptable after the bone healing period - and the boundary conditions. Materials are mostly treated as continuum, with material properties usually set homogenous and linear with elastic material properties characterized by two material constants: Young's modulus and Poisson's ratio. This 
assumption approaches the behaviour of the metal alloys quite well, the properties of the bone are still intensively investigated.

Bone mineral density, geometry of bone, microarchitecture of bone, and quality of the bone material are all components that determine bone strength as defined by the bone's ability to withstand loading. For this reason, microstructural information must be included in the analysis to predict individual mechanical bone properties [17].

Microstructural modelling of trabecular bone has become an extensively investigated field of biomechanical researches nowadays. The most commonly used tool for characterizing the complex architecture and material properties of bony organs is the conversion of micro-computed tomography images into micro-FE models. The basic assumption of the conversion is that the spatial distribution of material properties, mass density values and Young's modulus follow the distribution of Hounsfield Units, representing the X-ray attenuation of the tissues [4].

The micro-computed tomography images (3D high resolution images) are transformed into a finite element model by the means of different methods. The 3D reconstruction can be directly transformed into an equally shaped micro finite element model by simply converting all voxels to equally sized 8-node brick elements (identical hexahedral elements) (Fig. 2). This results in finite element models with a very large number of elements, which means the need of high computational capacity and time [16]. Since cancellous bone in the jaw-bones can be considered as an open-celled cellular structure, computational time can be reduced by representing each trabecula with just one beam element, which leads to the reduction in computational efforts as well [9] (Fig. 2). In this method each rod is modelled with one thickness-matched beam. The connection points as determined from the topological classification form the nodes for each beam [9]. The thus achieved results are less accurate at the local stress fields, but detailed enough to estimate the overall mechanical properties.

The afore-mentioned methods have been developed for simulating the mechanical behaviour of several types of bones of the human skeleton, usually for simulating the effect of bone diseases - such as osteoporosis - on the mechanical properties of the bone. In the majority of cases the models are from the vertebral or the femoral bone substance and not from the mandible or maxilla and include no implants or cortical layer in or around the examined trabecular bone.

The purpose of this study was to create a new, numerical microstructural bone modelling method that promotes the further investigations of the bone tissue surrounding dental implants and - combined with finite element modelling of screwtype implants - is applicable for simulating the mechanical behaviour of the cancellous bone and produces mechanical properties comparable to that of the real bone tissue, without the use of CT imaging. Striving for simple and fast modelling a beam model was created in a stochastic way to avoid the need of CT imaging.
The received microstructural frame model of the trabecular bone substance is planned to be combined with cortical layer around and implant imbedded in it, to examine the behaviour of the bone surrounding implants with different geometric conformations during their normal function after the bone healing.

\section{Methods and results}

The afore-mentioned bone models applying either volumeor beam-elements (Fig. 2) have their limits in the use of CTimaging. In micro-computed tomography imaging cadaveric samples are examined. In conventional CT-imaging or in Cone Beam Computed Tomography (CBCT) or Dental Volume Tomography (DVT) scanners, which are the latest generations of CT imaging in dentistry the - often otherwise healthy - patients are exposed to radiation exposure. On the contrary densitometry is accompanied by negligible radiation dose. Furthermore these kinds of examinations are patient specific and only suitable for describing the small, previously scanned fragment of the bone. The purpose of our research was to create a numerical model of the trabecular bone substance, which requires no CT-imaging as input data.

The initial objective was to create a program that generates a stochastic beam structure, which has the format identical with the input data of program system ANSYS - a general purpose finite element modelling system for numerically solving a wide variety of mechanical problems - and has parameters, which are revisable according to the bone that is simulated (in the aspect of density, porosity, elastic properties etc.). In this stage of the researches a finite element bone model was produced, which on the microstructural level - possesses the properties of the trabecular bone substance of an average man from the edentulous mandibular region, such as the geometry (average length and diameter) and the material attributes of a single tracecula or the porosity (or density) of the bone substance. In this study the model was submitted to compression and was modified in several - later expounded - ways to achieve the best conceivable global elastic properties. Other types of loading such as shearing and torsion and the effect of varying bone density require further examinations.

\subsection{Generating a model}

The finite element beam model of the cancellous bone is created by interlinking a stochastically generated set of nodes in a certain domain (Representative Volume Element), according to a previously defined linking-rule. The extent of the Representative Volume is characteristic of the mechanical properties of the modelled material volume and has to be selected to be convenient to the aims of the planned investigations.

In the present case the desired number of nodes was set in a $5 \mathrm{~mm} \times 5 \mathrm{~mm} \times 5 \mathrm{~mm}$ sized cubical shaped domain, which represents the identical sized bone substance. The stability of the model is provided by the appropriate number of linkings between the nodes and by the rigidity of the connections. Each 
Fig. 2. The micro-computed tomography reconstruction and the finite element mesh with hexahedral (up) and beam (down) elements of the trabecular bone substance, [www.manufacturingcenter.com/dfx/archives/0904/0904 micro.asp], [9]

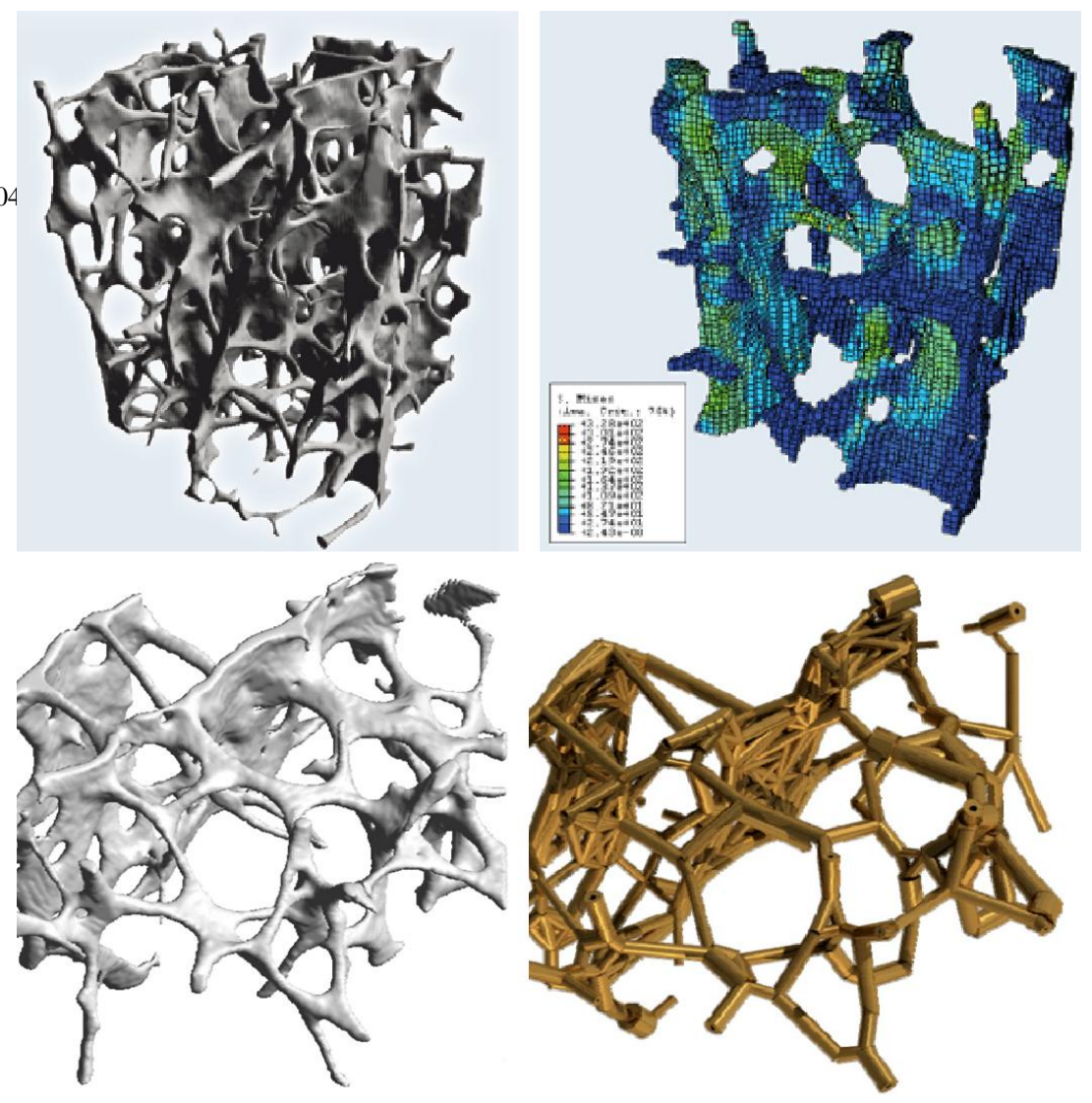

node is connected with a beam to the closest at least three nodes, but considering the trabecular bone of the human mandible this number has to be set from 5 to 7 . In the thus received finite element model each trabecula is represented by one beam element (Fig. 3). From the assortment of program system ANSYS the so-called BEAM188 element was applied, which is a three dimensional linear finite strain beam based on Timoshenko beam theory, in which shear deformation effects are included [20] (Fig. 4). The consideration of the shear deformations are justified because of the beams being slightly stubby and possessing porous contexture. As we mentioned before the micro-structural parameters - observable in Table 1 - were set according to that of the human mandibular bone in the following way.

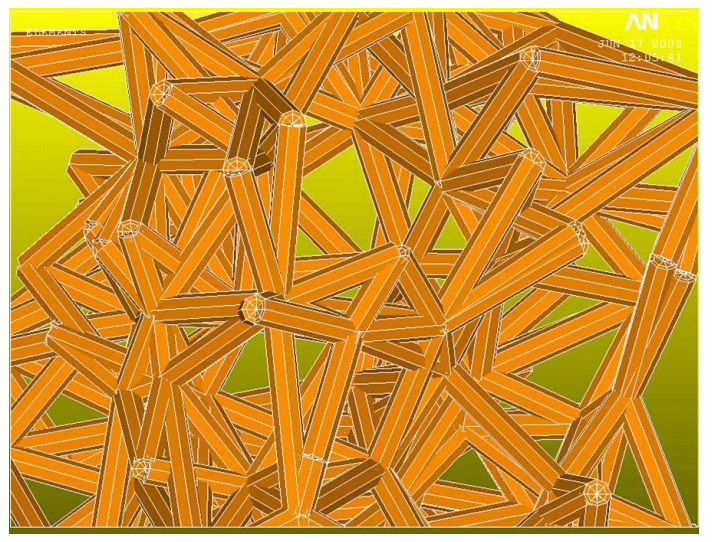

Fig. 3. Stochastically generated finite element frame model of the trabecular bone
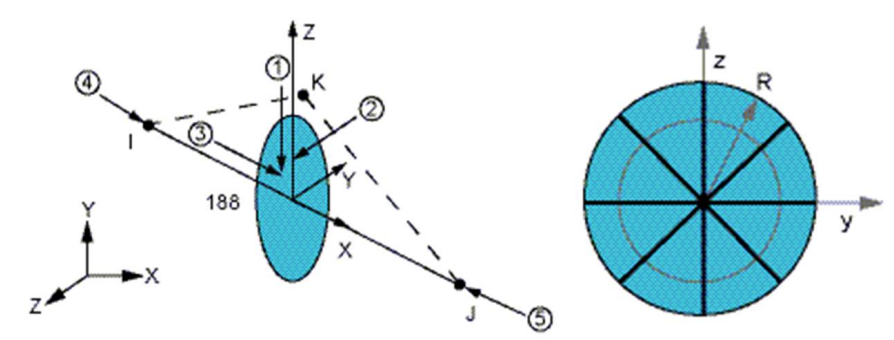

Fig. 4. The geometry of BEAM188 element from program system ANSYS and the applied cross section [20]

\subsection{Identification}

In the aforementioned $5 \mathrm{~mm} \cdot 5 \mathrm{~mm} \cdot 5 \mathrm{~mm}$ sized cubic domain 4000 nodes were placed in a stochastic way each of which was linked to its 7 closest neighbours. To this geometric conformation belongs $315 \mu \mathrm{m}$ average length of the trabeculae, which using $80 \mu \mathrm{m}$ beam diameter results in $70,4 \%$ porosity value (Table 1). To achieve the required approximately $70 \%$ porosity (percentage value of voids volume of bone material in proportion to the volume of the whole cube) - according to literary data [3, 5, 10, 13] - the number of the nodes and the applied linkings were changed, with the length and diameter kept on the abovementioned values. The trabeculae themselves are considered to have circular cross-section, to be lineal and have elastic material behaviour characterized by two micro material constants of Young's modulus and Poisson's ratio, the values of which are also drawn upon the literature (Table 1 ).

There have been several attempts to determine the Young's 
Tab. 1. The microstructural parameters of the examined frame model

\begin{tabular}{lc}
\hline Bone substance & $5 \mathrm{~mm} \times 5 \mathrm{~mm} \times 5 \mathrm{~mm}$ \\
Number of nodes & 4000 \\
Number of connections & 7 \\
Average beam length & $315 \mu \mathrm{m}$ \\
Beam diameter & $80 \mu \mathrm{m}$ \\
Porosity & $70.4 \%$ \\
Young modulus & $15 \mathrm{GPa}$ \\
Poisson ratio & 0.3 \\
\hline
\end{tabular}

modulus of the trabeculae using number of different techniques [3. 14]: direct mechanical testing of a single trabecula in tension (Ryan and Williams, 1989), 3-point bending (Kuhn et al., 1987 and Choi et al., 1990) or buckling (Townsend et al., 1975 and Runkle and Pugh, 1975); ultrasonic wave propagation and scanning acoustic microscopy in trabecular bone specimens (Ashman and Rho, 1988 [1]; Turner et al., 1999 [18]; and Nomura et al, 2007 [11]); finite element analysis (Pugh et al., 1973; Williams and Lewis, 1982; Mente and Lewis, 1987; van Rietbergen et al., 1995 and Kabel et al., 1999 [7]) and nanoidentation (Rho et al., 1997 [15]; Zysset et al., 1999 [19]). According to the results of these, the elastic modulus and Poisson's ratio of the single trabeculae were set $15 \mathrm{GPa}$ and 0,3 (Table 1 ).

\subsection{First tests of the model}

The received finite element frame model was submitted to compression tests with loads and boundary conditions observable in Fig. 5, and the macro-structural elastic properties were computed from the result data obtained by the means of program system ANSYS. The cubic shaped substance was supported at its lower side with hinged connection and no supports were applied on the side-nodes, thus simulating the boundary conditions of a mechanically tested bone specimen. The macro-structural elastic properties were calculated from the stresses arising from the displacement loads acting on the upper side of the cube and were required to be identical with the values reported in the literature. The obtainable literary Young's modulus values of cancellous bone specimens were estimated between 0,5 and $5 \mathrm{GPa}$ by the means of three different methods: using Hodgkinson and Currey's (1992) empirical equations relating modulus and density [3], by the means of finite element analysis [7] or compression tests [8, 12], according to which the results of the tested model had to be between 0,5 and $5 \mathrm{GPa}$. In the test $0,1 \mathrm{~mm}$ vertical displacement load was applied that means a $2 \%$ deformation in the cube. In calculating the macro-structural elastic modulus small deformations were assumed. The simulation produced the unsatisfying value of $0,347 \mathrm{GPa}$ showing that the model did not possesses the required stiffness (Fig. 6). The anatomic architecture of bone tissue had to be estimated more accurately. The forthcoming four attempts have been made to increase stiffness.

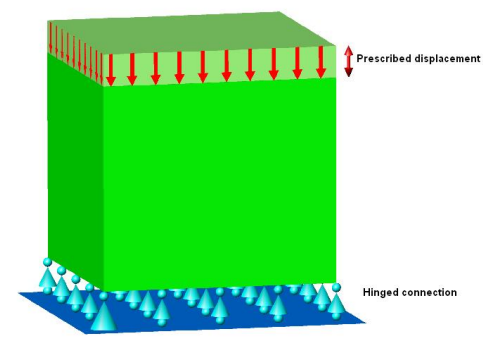

Fig. 5. Loads and boundary conditions in the compression test

\subsection{Increasing the overall stiffness}

The first attempt to achieve more accurate elastic properties was changing the boundary conditions according to that of a real bony cube imbedded in the neighbouring bone substance. The boundary nodes on the four sides were supported elastically in the two horizontal directions. The behaviour of the beam structure was examined using a wide range of elasticity values on these supports up to the almost rigid connection, which led to $25 \%$ increase in the elastic modulus at the most. While being mainly compressed, the changes in the inertias of the beam elements did not have a remarkable effect on the elastic properties either (Fig. 8). The third and much more effective method for developing the beam structure in the aspect of Young's modulus was the abolishment of its isotropy. Bone tissues have the ability to adapt their strengths to the applied loads. As other biological tissues, they are in continuous remodelling. Bone cells respond to local deformations of the bone produced by mechanical stress. With slightly increased strain, the bone becomes mildly overloaded and compensates by strengthening. The strain exceeding the allowable threshold causes fracture, but under a certain level results in bone desorption [6]. To simulate this phenomenon, elastic modulus of each and every beam was changed as a function of the structural forces arisen in them - under the average value of the forces decreased, and above increased with a ratio calculated according to the deviation from the mean value. In the thus received new orthotropic model significant stiffening was observable, which was however dependent on the rule according to which the beams were varied. This modification applied with unidirectional compression makes the developed method suitable for modelling the behaviour of more orientated cancelluos bone tissues than in jaw-bones, for example vertebral or femoral bone substances as well. While no reported studies could be found in the literature that would numerically describe the changes in the cancellous bone's micro-mechanical properties during functional adaptation, this model temporarily remains theoretical with no adjusted parameters, but simulating the tendency of the remodelling process. Although the load dependent, regular conformation of the trabeculea in jaw bone is not so clearly observable like in long bones, it still exists. In case the method of bone remodelling could be quantified, this method could be effectively applicable not only according to the 

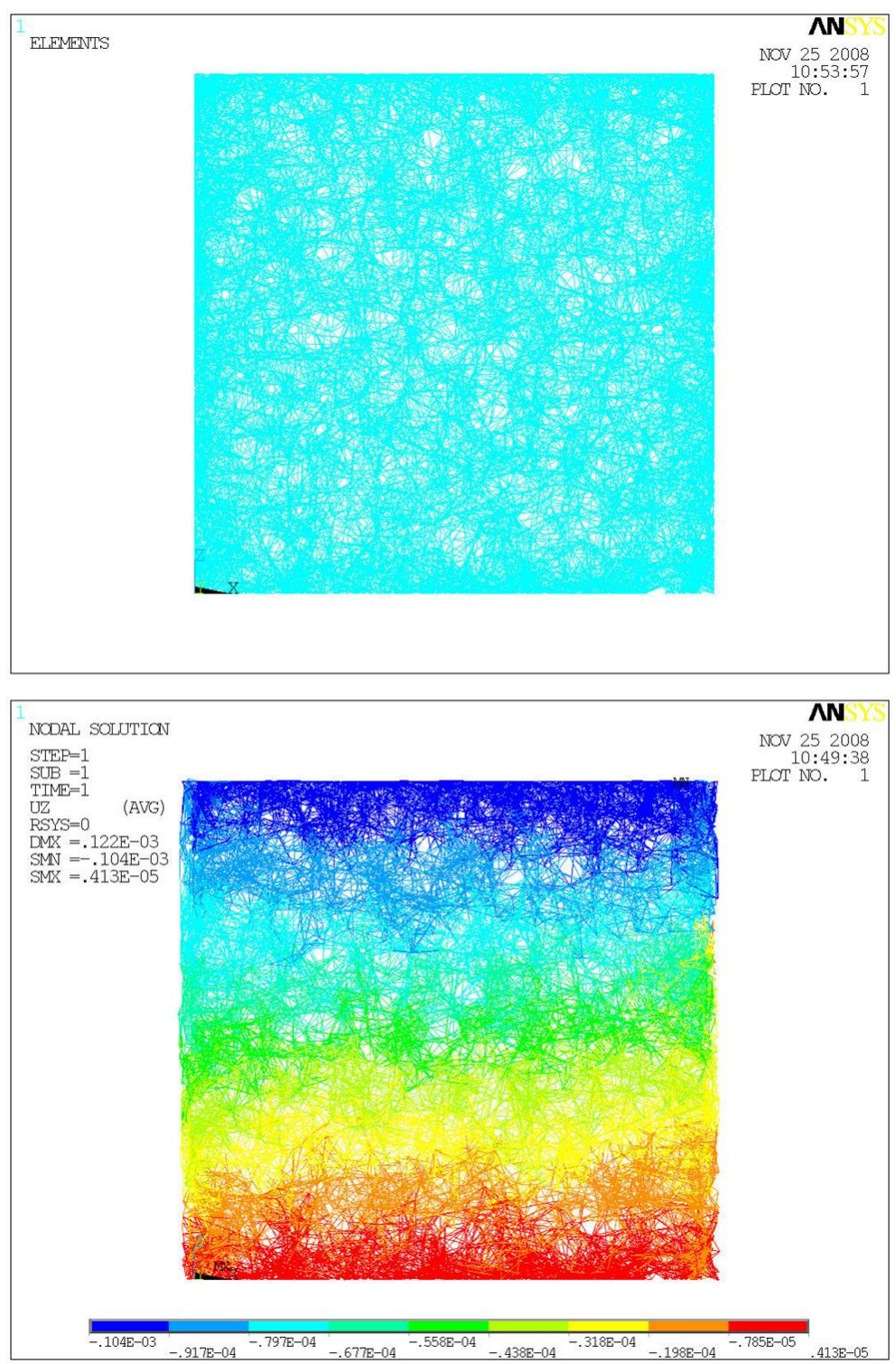

Fig. 6. The tested cubic shaped frame model (up) and its deformed shape with the vertical displacements from the compression test (down)

forces from unidirectional compression, but in further examined models as well, where the bone is combined with a finite element model of a screw type implant, which transmits the occlusal forces to the bone. Neither the original nor the aforementioned three modified models consider the shell behaviour of the trabeculae resulting from their anatomically bent geometry. In the aspect of a beam model this can be taken into account by using combined beam elements with higher stiffness values at the regions closer to the ends and lower in the middle. In the fourth and most expedient modified model each beam was divided into three parts with four orders higher (the lower bound that was considered infinitely stiff by the program system ANSYS) elastic modulus values at the ends, than at the middles, thus increasing the rigidity of the connections between the beam elements as well. Fig. 7 shows the construction of the modified beam elements. The central parts of the beams possess the original, aforementioned $15 \mathrm{GPa}$ Young's modulus data, in contradiction to the $15 \cdot 10^{4} \mathrm{GPa}$ values at both two ends of the beams. Applying this strengthened beam structure a 1,311 GPa global - macro-structural - Young modulus value was achievable (Fig. 8), which satisfactorily remains within the limits of the literary data of 0,5 and $5 \mathrm{GPa}$. In further examinations the thus received model has to be tested under other, physiological loads and then has to be completed with the oral implant imbedded in the cancellous bone substance.

\section{Conclusions}

The mechanical behaviour of biological materials - such as cancellous bone - are most commonly examined by the means of direct mechanical testing or finite element analysis, the latter of which is the in vivo applicable method in humans. In the aspect of oral implantation in the upper- and lower-jawbones the finite element models reported so far consider the trabecular bone substance as a continuum. The fact, that microstructural properties have remarkable effect on the overall behaviour of the bone, indicates the need of micromechanical simulations. The most commonly used method for modelling the cancellous bone's microstructure is the conversion of CT images into finite 
element meshes using either volume or beam elements, the latter of which overcomes the difficulties resulting from the high computational time and effort demand, while producing less accurate results. The application of CT imaging can be avoided by creating a stochastically generated, porosity (or density) dependent finite element frame model, in which each trabecula is simulated by three beam elements with different elastic properties at the two ends and in the middle. The geometrical and mechanical properties, such as the length and diameter of the trabeculae, the porosity and material properties of the bone can be changed according to the anatomic circumstances. According to the finite element - so far compression - simulations, the use of elastic supports at the side plates of the cubic substance and the modification of the beam inertias have no remarkable effect on the global elastic modulus of the examined domain. In contradiction to this the overall stiffness can be significantly increased by using load dependent anisotropy on the structure or by making the end regions of the beams several orders stiffer, than the middle, thus simulating the shell behaviour and the higher connection stiffness of the trabeculae. In further investigations the stochastically generated finite element beam model of the trabecular bone is planned to be tested under different types of loadings and combined with finite element dental implant models.

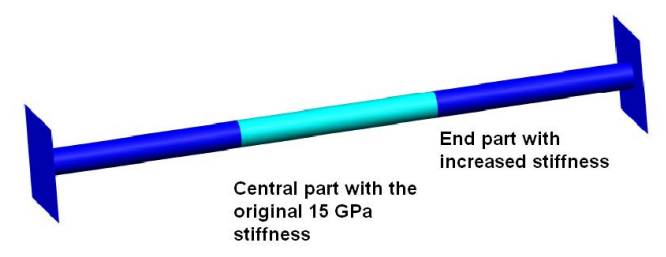

Fig. 7. The construction of the modified beam elements

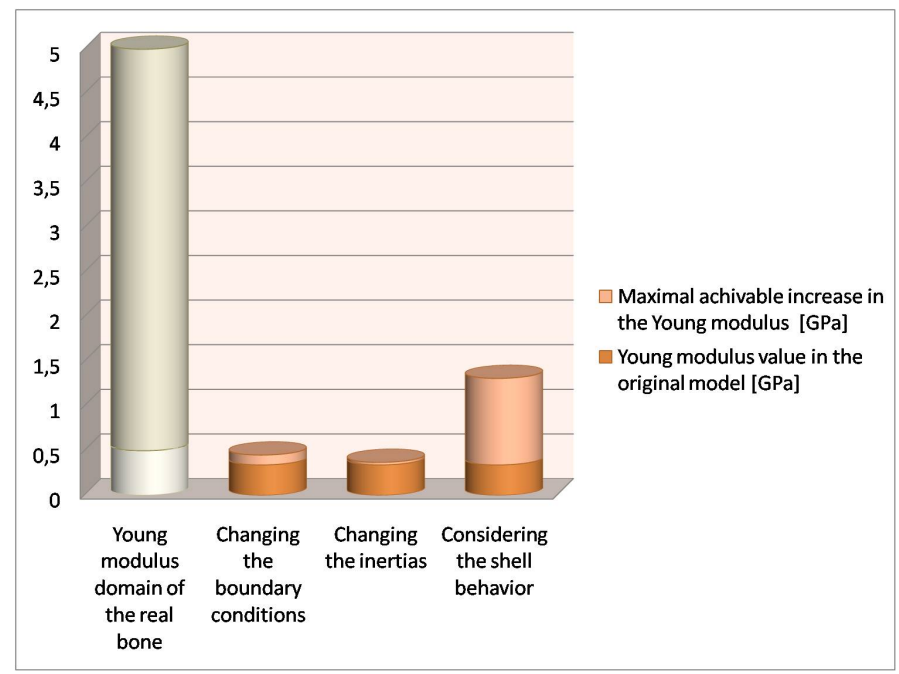

Fig. 8. The effectiveness of the three Young modulus increasing methods in the aspect of the stochastically generated frame model

\section{References}

1 Ashman RB, Rho JY, Elastic modulus of trabecular bone material, Journal of Biomechanics 21 (1988), no. 3, 177-181, DOI 10.1016/00219290(88)90167-4.
2 Divinyi T, Fogászati implantológia, Springer Hungarica, Budapest, 1998.

3 Gibson LJ, Ashby MF, Cellular solids - Structure and properties, University Press, Cambridge, 1997. second ed.

4 Hellmich C, Kober C, Erdmann B, Micromechanics-Based Conversion of CT Data into Anisotropic Elasticity Tensors, Applied to FE Simulations of a Mandible, Annals of Biomedical Engineering 36 (2008), no. 1, 108-122, DOI 10.1016/S0021-9290(08)70202-1.

5 Hogskinson R, Njehz CF, Whitehead MA, Langton CM, The non-linear relationship between BUA and porosity in cancellous bone, Phis. Med. Biol. 40 (1996), 2411-2420, DOI 10.1088/0031-9155/41/11/012.

6 Isidor F, Influence of forces on peri-implant bone, Clin. Oral. Impl. Res. 17 (2006), no. 2, 8-18, DOI 10.1111/j.1600-0501.2006.01360.x.

7 Kabel J, Van Rietbergen B, Dalstra M, Odgaard A, Huiskes R, The role of an effective isotropic tissue modulus in the elastic properties of cancellous bone, Journal of Biomechanics 32 (1999), 673-680, DOI 10.1016/S00219290(99)00045-7.

8 Kang Q, An YH, Friedman RF, Mechanical properties and bone densities of canine trabecular bone, Journal of Materials Science: Materials in Medicine 9 (1998), 263-267.

9 Van Lenthe GH, Stauber M, Müller R, Specimen-specific beam models for fast and accurate prediction of human trabecular bone mechanical properties, Bone 39 (2006), 1182-1189, DOI 10.1016/j.bone.2006.06.033.

10 Moon HS, Won YY, Kim KD, Ruprecht A, Kim HJ, Kook HK, Chung MK, The three-dimensional microstructure of the trabecular bone in the mandible, Surg. Radiol. Anat. 26 (2004), 466-473, DOI 10.1007/s00276004-0247-x.

11 Nomura T, Katz JL, Powers MP, Saito C, A micromechanical elastic property study of trabecular bone in the human mandible, J. Mater. Sci.: Mater. Med. 18 (2007), 629-633, DOI 10.1007/s10856-007-2312-6.

12 O'Mahony AM, Williams JL, Katz JO, Spencer P, Anisotropic elastic properties of cancellous bone from a human edentulous mandible, Clinical Oral Implants Research 11 (2000), no. 5, 415-421, DOI 10.1034/j.16000501.2000.011005415.x.

13 Renders GA, Mulder L, Van Ruijven LJ, Van Eijden TMG J, Porosity of human mandibular condylar bone, J. Anat. 210 (2007), no. 3, 239-248, DOI 10.1111/j.1469-7580.2007.00693.x.

14 Rho JY, Kuhn-Spearing L, Zioupos P, Mechanical properties and the hierarchical structure of bone, Medical Engineering \& Physics 20 (1998), 92-102, DOI 10.1016/S1350-4533(98)00007-1.

15 Rho JY, Tsui TY, Pharr GM, Elastic properties of human cortical and trabecular lamellar bone measured by nanoindentation, Biomaterials 18 (1997), no. 20, 1325-1330, DOI 10.1016/S0142-9612(97)00073-2.

16 Rudnyi EB, Van Rietbergen B, Korvik JG, Efficient harmonic simulation of a trabecular bone finite element model by the means of model reduction, Proc. 12th Workshop "The Finite Element Method in Biomedical Engineering, Biomechanics and Related Fields", July 2005, pp. 61-68. Proceedings of the 12th FEM Workshop.

17 Stauber M, Müller R, Volumetric spatial decomposition of trabecular bone into rods and plates - a new method for local bone morphometry, Bone $\mathbf{3 8}$ (2006), 475-484, DOI 10.1016/j.bone.2005.09.019.

18 Turner $\mathbf{C H}$, Rho JY, Takano Y, Tsui TY, Pharr GM, The elastic properties of trabecular and cortical bone tissues are similar results from two microscopic measurement techniques, Journal of Biomechanics 32 (1999), 437-441, DOI 10.1016/S0021-9290(98)00177-8.

19 Zysset PK, Guo XE, Hoffler CE, Moore KE, Goldstein SA, Elastic modulus and hardness of cortical and trabecular bone lamellae measured by nanoindentation in the human femur, Journal of Biomechanics 32 (1999), 1005-1012, DOI 10.1016/S0021-9290(99)00111-6.

20 ANSYS 11.0 User's Manual. 